\title{
Impact of surfactant addition on non-Newtonian fluid behavior during viscous fingering in Hele-Shaw cell
}

\author{
Seyedarash Ahmadikhamsi, ${ }^{1}$ Fabrice Golfier, ${ }^{1}$ Constantin Oltean, ${ }^{1}$ Eric Lefèvre ${ }^{1}$ and S. Amir Bahrani, ${ }^{2, a)}$ \\ ${ }^{1}$ Université de Lorraine, GeoRessources, UMR 7359 CNRS, Nancy F-54000, France \\ ${ }^{2}$ IMT Lille Douai, Univ. Lille, Energy Engineering Department, F-59000 Lille, France
}

\begin{abstract}
We present an experimental study of viscous fingering caused by the displacement of an oil phase by non-Newtonian fluids such as Carbopol ${ }^{\circledR} 940$ with and without surfactant (SDS) addition in a radial Hele-Shaw cell. When polymer solutions are injected, a variety of fingering patterns as a function of flow rate are observed, which differ from the classical Saffman-Taylor (ST) instability. We have shown that if surfactant concentration locally decreases the interfacial tension, it also leads -to a reduction of viscosity and hence results in an increasing impact on capillary number. We found that surfactant-polymer solutions have wider fingers with increasing flow rates in contrast with Newtonian solutions. Our study also revealed that the relative finger width of both non-Newtonian experiments with and without surfactant converge asymptotically to the same value. We think that this phenomenon is caused by the decrease of surfactant concentration in the vicinity of the tip as the finger is growing so that the shear-thinning features of polymer prevail at long time.
\end{abstract}

\section{INTRODUCTION}

During the last decades, an increasing attention in oil industry has been given to the use of polymer-surfactant solutions to control hydrodynamic instabilities during Enhanced Oil Recovery (EOR) ${ }^{1,42}$. Such a recovery technique is generally applied when the mobility ratio is unfavorable or when the reservoir is heterogeneous (even with favorable mobility ratio) to recover bypassed oil ${ }^{2}$. Indeed, one of the most important disadvantages of EOR processes is the development of so-called viscous fingering ${ }^{43}$ as it results in preferential channeling which may affect the reservoir oil production. The study of viscous fingering has a long story going back to the pioneering work of Saffman and Taylor in $1958^{3}$. The authors reported the occurrence of instability and fingering-like pattern at the interface between two fluids confined in a flow cell or Hele Shaw cell, when a less viscous fluid is injected into a more viscous fluid. While capillary forces play a notably different role in porous media where the two phases coexist within the saturation front zone at the microscopic scale ${ }^{44,45}$, the Hele Shaw experiments have helped to give physical insight into the onset of viscous instabilities. Different types of Hele Shaw cell geometry (rectangular or radial for investigating competition between multiple fingers) have been used historically for this purpose ${ }^{4-7}$. In the absence of buoyancy effect and with a constant cell aspect ratio, it was found for immiscible fluids that the emergent fingering patterns are mainly driven by the rheological characteristics of fluids through the mobility ratio and the capillary number $\mathrm{Ca}$, defined as the ratio between viscous and capillary forces. In this study $\mathrm{Ca}$ is defined as

a) Electronic mail: amir.bahrani@imt-lille-douai.fr 


$$
C a=V \Delta \mu / \sigma
$$

where $V$ is a characteristic velocity, $\Delta \mu$ is the difference of dynamic viscosity between both immiscible fluids and $\sigma$ is the interfacial tension between displaced and displacing fluid.

To improve the sweep efficiency of the conventional waterflood and balance the negative impact of viscous fingering, recent studies suggest the use of chemical injections as polymer-surfactant solutions. The large-molecular-weight and high-viscosity polymer increases the viscous forces by promoting the oil displacement while the surfactant solution reduces the capillary forces of the fluid in place ${ }^{8-10}$. Nevertheless, the non-Newtonian fluids exhibit different behaviors like shear thinning, yield stress and elasticity effects which may dramatically impact viscous fingering pattern morphology due to spreading, side branching and splitting processes ${ }^{10-14}$. The classical Saffman-Taylor theory is not valid anymore since additional flow features need to be considered through the use of Weissenberg, Weber or Bingham dimensionless numbers for instance. This has motivated a significant research effort on non-Newtonian fluids during the last decades ${ }^{15,16}$. Lindner et al. ${ }^{8,9}$ have investigated the impact of non-Newtonian properties separately. Narrower fingers have been observed for shear-thinning fluid due to viscosity decrease along the finger in the flow direction. On the contrary, wider fingers caused by elongational viscosity are found for visco-elastic fluids. Impact of shear-thickening properties on finger width has been also considered ${ }^{9,10}$. Different patterns with a single or multiple ramified fingers have been identified by Lindner et al. ${ }^{8}$ for yield stress fluids. The existence and competition of these various flow regimes have been generalized for a complex elasto-visco-plastic non-Newtonian fluid ${ }^{15}$. Eslami et al. ${ }^{16}$ also reported that wettability conditions at the walls of the Hele-Shaw cell may affect fingering dynamics and display different flow regimes, from a comparative analysis between wetting displacements (oil-Carbopol) and non-wetting displacements (air-Carbopol).

Surfactant impact on viscous fingering patterns for water/oil or water/air system has also received a great deal of attention. Several authors ${ }^{8,13,18,19}$ observed that the reduction of dynamic interfacial tension induced by adding surfactant to a Newtonian fluid causes finger widening in contrast with the Saffman Taylor theory. According to Lindner et al., ${ }^{8}$ Bonn et al., ${ }^{13}$, this is due to a heterogeneous distribution of surfactant molecules at the finger tip which yields an anisotropy of the surface tension. Recently Tsuzuki et al. ${ }^{19}$ proposed an alternative explanation and suggested that surfactant advection could be enhanced by a combination of fountain flow and Marangoni effect ${ }^{46}$. Niroobakhsh et al. ${ }^{20,21}$ investigated the injection of cationic surfactant solution into oleic acid and included the interfacially forming material as a cause of the viscous instabilities.

Even if a number of studies have investigated the impact of fluid rheology or surfactant addition on viscous fingering, very few have considered experimentally the combination of both at the notable exception of Yamamoto et al., ${ }^{11}$ and Bonn et al., ${ }^{12}$. In these two research papers, highly concentrated surfactant solutions with micelle structures, similar to a polymer solution and exhibiting shear-thinning and antithixotropic features, were considered. These surfactant solutions have shown anomalous patterns such as needle-like fingering under certain conditions.

None of these works, however, have investigated the modifications induced by surfactant addition on polymer rheology and the consequences on viscous fingering. Certain polymers in the presence of surfactant solutions, indeed, exhibit microstructural interactions that may affect the rheological properties of the solution (mainly the shear-dependent viscosity) and, hence, the fingering instabilities ${ }^{22}$. Various studies have characterized the physical nature of water soluble polymers - surfactant systems 
and several factors influence the interactions between surfactant and polymers such as the addition of electrolyte, the type of surfactant chain and structure, polymer molecular weight and concentration, surfactant and polymer adsorption, intermolecular interactions, colloidal stability, micellization effects and the respective rheological behaviors of polymers and surfactants in solution $^{23-28}$. The viscosity of hydrophilic polymers is thus found to be very sensitive to surfactant concentration ${ }^{29-32}$. Milanovich et al. ${ }^{31}$, for instance, have compared the effect of different concentrations of anionic surfactant (SDS) and nonionic surfactant (Tween80) on the structure of Carbopol 940 polymer in aqueous solution. They revealed that the surfactant-induced structural changes were the result of hydrophobic association between surfactant micelles and Carbopol 940 polymer tails.

As a conclusion, the goal of this study is to experimentally investigate the combined impact of the modified fluid rheological properties and surface tension reduction induced by surfactant additive on a non-Newtonian polymer solution in the development of viscous fingering patterns. An anionic surfactant and a complex yield stress shear-thinning fluid will be considered in the current work. Viscous fingering patterns obtained for the same non-Newtonian fluid in the absence of surfactant and the classical Saffman-Taylor finger-like patterns will be also presented and analyzed for comparative purpose.

\section{MATERIAL AND METHODS}

\section{A. Rheological properties of fluids used}

The working fluids used in this study are: $(i)$ a silicone oil as continuous phase which is a Newtonian fluid with the viscosity of 5 (Pa.s), and (ii) a $95.5 \mathrm{wt} \%$ Glycerol solution (Newtonian reference fluid) and a $0.12 \mathrm{wt} \%$ aqueous solution of Carbopol ${ }^{\circledR}$ 940 (polyacrylic acid polymer) as dispersed phase. In order to control the rheological and surface tension of Carbopol solutions three different concentrations (0.01, 0.05 et $0.1 \mathrm{wt} \%$ ) of Sodium Dodecyl Sulfate (SDS), as anionic surfactant agent, are used. The Carbopol polymer is dissolved in distilled water during 2 hours and then is left to rest for 24 hours ${ }^{33}$. The Carbopol dispersion is acidic $(\mathrm{pH} \approx 3)$ and neutralized by adding $10 \%$ of solution of sodium hydroxide $(\mathrm{NaOH})$. The final aqueous solution is in the form of a translucent gel. It is left to rest for 48 hours before conducting the rheological tests. Rheological data were obtained using an Anton-Paar MCR 302 rheometer with cone-plate geometry $\left(50 \mathrm{~mm} / 1^{\circ}\right)$. The rheological measurement protocol was as follows: first the fluid is sheared at $5 \mathrm{~s}^{-1}$ during $5 \mathrm{~min}$ and then kept to rest during $3 \mathrm{~min}$ so that the material restructures ${ }^{33}$. Subsequently a shear-rate range (including the range of shear rates investigated within the HeleShaw cell), $0.1 \leq \dot{\gamma} \leq 1000 \mathrm{~s}^{-1}$, is applied with the rheometer to describe the flow behavior. 

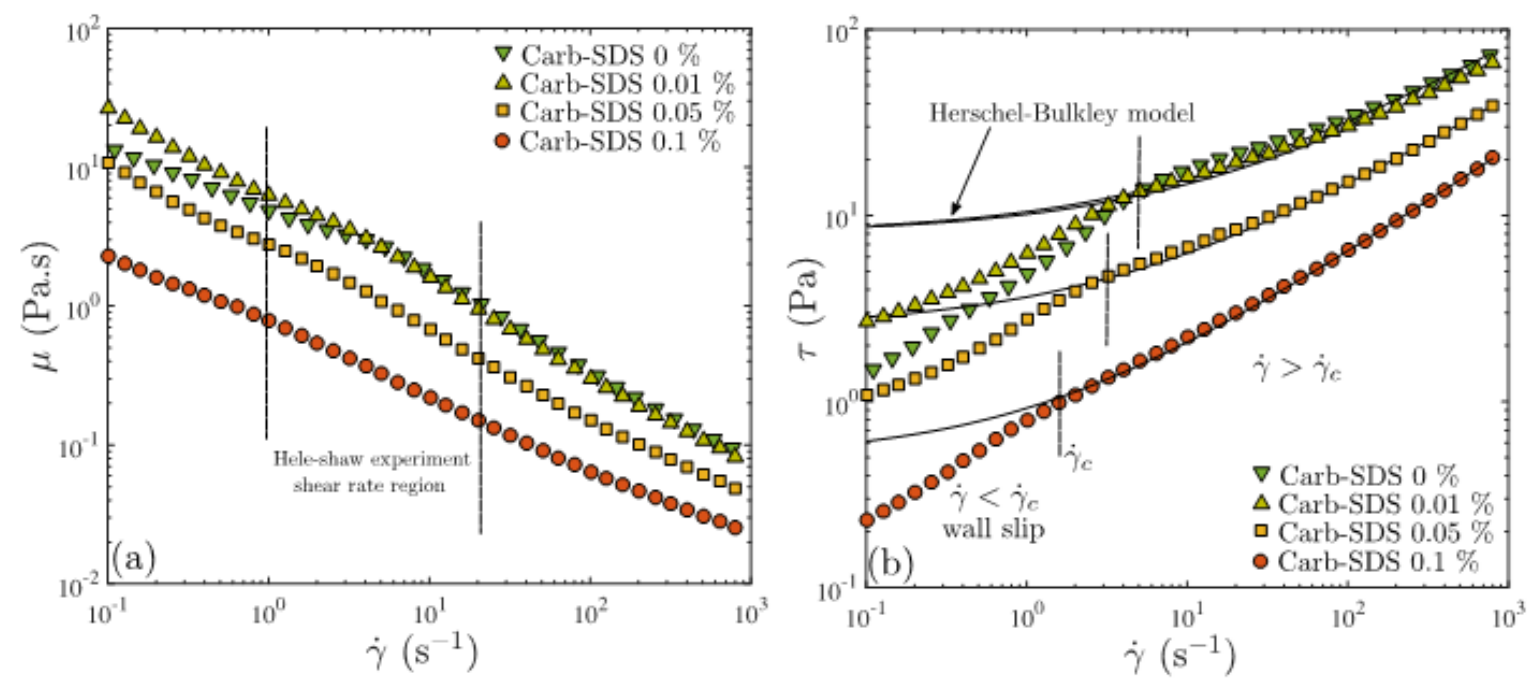

FIG. 1. Flow curves of the Carbopol solutions with different SDS concentrations obtained from rheological measurement. (a) viscosity vs shear rate and (b) stress $v s$ shear rate. The continuous line represents the Herschel-Bulkley model applied to flow curves.

The flow curves obtained (Fig. 1) are characterized by fitting the Herschel-Bulkley model, $\tau=\tau_{0}+K \dot{\gamma}^{n}$, at the region $\dot{\gamma}>\dot{\gamma}_{c}$. Where $\tau, \tau_{0}, K$ and $n$ are the shear stress, yield stress, consistency and power law index, respectively. We have clearly identified two regions (Fig. 1b), separated by a critical shear rate $\dot{\gamma}_{c}$, that changed with SDS concentration. We show that for $\dot{\gamma}<\dot{\gamma}_{c}$ a wall slip occurs. This behavior is due to non-homogeneity of the shear stress distribution across the gap of coneplate rheometer. This region can be characterized by a competition between destruction and reformation of the gel network ${ }^{34}$.

The rheological parameters for Carbopol solutions are summarized in Table I. The results show that the rheological properties of pure Carbopol solution are quasi-similar to the ones obtained for $0.01 \%$ SDS concentration but differ beyond this value. The yield stress for $0.12 \mathrm{wt} \%$ Carbopol solution is $7.9 \mathrm{~Pa}$ and decreases when increasing the SDS concentration. A similar tendency is also observed for consistency index contrary to power law index since the values of $n$ increase with increasing surfactant concentration. This behavior is due to role of surfactant agent as viscosity reducer. For the range of concentration under study, indeed, the interaction of SDS with Carbopol microgels induces an increase of polymer ionic strength accompanied by gel matrix shrinking, resulting in a decrease in the apparent viscosity of the solution. Note that for higher concentrations (>0.1\% SDS), an opposite effect occurs: the increase of osmotic pressure causes the matrix swelling and thus viscosity increases. Above the SDS binding concentration $(0.6 \%)$, viscosity decreases again because of the presence of free micelles ${ }^{40}$.

TABLE I. Rheological parameters, surface tension and density of the Glycerol and Carbopol solutions with different SDS concentrations.

\begin{tabular}{cccccc}
\hline \hline Sample & $\tau_{0}$ & $K$ & $n$ & $\gamma$ & $\rho$ \\
& $P a$ & $P a . s^{n}$ & & $m N . m^{-1}$ & $k g . m^{-3}$ \\
\hline \hline Glycerol 99.5\% & - & - & - & 30.3 & 1259 \\
\hline Carb-SDS 0.0 & 7.9 & 2.56 & 0.48 & 36 & $999.3 \pm 0.3$ \\
Carb-SDS 0.01 & 7.5 & 2.44 & 0.48 & 20 &
\end{tabular}




$\begin{array}{ccccc}\text { Carb-SDS 0.05 } & 2.5 & 1.1 & 0.52 & 16 \\ \text { Carb-SDS 0.1 } & 0.2 & 0.5 & 0.54 & 13\end{array}$

As can be seen in Fig. 1a we also defined the shear-rate region in which Hele-Shaw experiments occur, i.e. $1.44 \leq \dot{\gamma} \leq 28$ $\mathrm{s}^{-1}$. For this range of shear rate, it should be noted that the contribution of the yield stress term cannot be neglected. The shear rate is not so high that the fluids behavior degenerates towards a purely power-law model. However, based on the analysis of different flow regimes of Carbopol conducted by Eslami et al. ${ }^{15}$, it appears that our experiments are not in the yield stress regime, characterized by the independency of finger width relative to the finger tip velocity ${ }^{9,15}$, but rather in the viscous regime. This suggests that the shear-thinning features of Carbopol should drive viscous fingering. We will discuss further this point in the Results and Discussion section.

Note that weakly elastic properties have been also reported in the literature for our Carbopol concentration ${ }^{35}$. The elastic properties can be characterized through the first normal stress difference, $N_{l}$, that becomes significant from $\dot{\gamma}>100 \mathrm{~s}^{-1}$ for the fluids of interest in this work. Therefore we neglected the elastic effects of Carbopol solution in the range of shear rate encountered in our experiments.

\section{B. Surface tension measurement}

Surface tension measurements were performed using the pendant drop method ${ }^{36}$ shown in Fig. 2. In order to perform each experiment, a drop was formed in an open cuvette that was prefilled with silicone oil. A syringing pump is used for injecting the fluid until a desired drop size was achieved and then recorded using a CCD camera connected to a PC. Afterwards the surface tension was calculated via a homemade Matlab $^{\circledR}$ code.

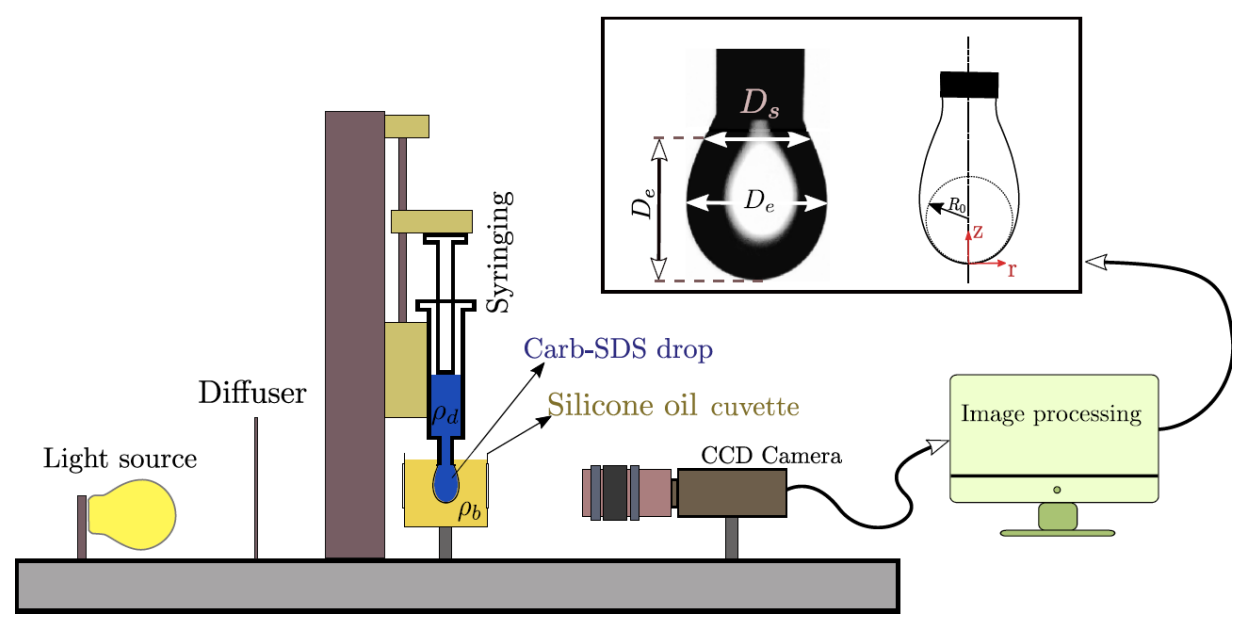

FIG. 2. Sketch of the pendant drop experimental apparatus. 
The interfacial tension is then calculated from the Bond number as follows ${ }^{1}$ :

$$
B o=\frac{\Delta \rho g R_{0}^{2}}{\gamma}
$$

where the density difference is $\Delta \rho=\rho_{b}-\rho_{d}, \rho_{b}$ and $\rho_{d}$ are the bulk (silicone oil) and drop (Carb+SDS) densities, respectively. $R_{0}$ is the drop dimension, $\gamma$, the interfacial tension and $g$, the gravitational acceleration. Firstly, for system calibration purpose, the value of surface tension was measured for $95.5 \mathrm{wt} \%$ glycerol as droplet phase and silicone oil as continuous phase and we obtained $30.3 \pm 0.41 \mathrm{mN} . \mathrm{m}^{-1}$. Similar measurements were performed for pure Carbopol and aqueous mixed solution of Carbopol $(0.12 \%)$ and $\operatorname{SDS}(0.01,0.05$ and $0.1 \%)$ in contact with silicone oil as continuous phase. The results are summarized in Table I. The value of surface tension for pure Carbopol (Carb-SDS 0) was measured of about $36 \pm 0.34 \mathrm{mN}^{-1}$ and then reduced significantly when increasing the SDS concentration to reach a value close to $13 \pm 0.38 \mathrm{mN} . \mathrm{m}^{-1}$ for $0.1 \%$ SDS concentration (Carb-SDS 0.1).

This combined reduction of viscosity and surface tension induced by surfactant addition leads to a enhanced effect on the capillary number. As an example, for a given velocity and assuming a constant shear stress and the same invaded fluid (silicon oil), adding $0.1 \%$ SDS concentration to Carbopol solution leads to an increase of about a factor of 4 of capillary number. Hereafter, we will focus only on the lowest SDS concentration for which the impact on viscosity is practically indiscernible so that we may study separately the impact of the change in surface tension on polymer behavior. In other words, we will investigate the viscous fingering patterns resulting from injection of two non-Newtonian fluids (Carb-SDS 0 and Carb-SDS 0.01) with the same rheological behavior but different surface tension.

\section{Experimental set-up and procedure}

Experiments were performed in a radial Hele-Shaw cell (see Fig. 3a). The cell consists of two rectangular parallel transparent plates disposed horizontally and placed $0.25 \mathrm{~mm}$ apart $(b)$. The plates are made of polished glass $300 \mathrm{~mm}$ in length $(L), 200$ mm width $(l)$ and $10 \mathrm{~mm}$ thickness $(e)$. The constant gap between the two plates is ensured by four Plexiglas triangular spacers $0.25 \mathrm{~mm}$ thickness $( \pm 2 \%)$ disposed at each corner of the cell. A $2 \mathrm{~mm}$ diameter injection hole was drilled in the center of the lower plate and a syringe pump (Model $100 \mathrm{KD}$ Scientific, New Hope, PA, USA) is connected via plastic tubing to the hole. Initially, the cell is saturated with silicone oil that is the more viscous fluid. The less viscous fluid (Glycerol or Carbopol solutions with or without surfactant addition) is injected into the cell with fixed flow rates ranging from 3.5 to $10 \mathrm{ml} / \mathrm{h}$. The gap at the edges of the cell is maintained open so that we have a boundary condition of constant atmospheric pressure along the external borders. The experimental tests were carried out at ambient temperature $\left(\mathrm{T} \approx 22{ }^{\circ} \mathrm{C}\right)$. A blue dye was mixed with the injection solution to visualize finger propagation and the high contrast obtained leads to a robust thresholding of the images (see the next section). No effect of the dye concentration on fluid properties was found. A standard CCD camera (EOS 7D

\footnotetext{
1 If we know $D_{e}$ and $D_{s}$ the maximum diameter of the drop and the diameter of the drop at a distance $D_{e}$ from the top, respectively, we have $\sigma=D_{s} / D_{e}$ and then $R_{0}$ is calculated by $R_{0}=D_{e}\left(0.99+0.19 \beta-0.07 \beta^{2}+0.34 \beta^{3}\right) / 2$ with $\beta=0.12-0.75 \sigma+1.77 \sigma^{2}-0.54 \sigma^{3}$. If we know fluids density $(\Delta \rho)$, finally the surface tension $(\gamma)$ is obtained from Eq. $2(\beta \equiv B o)$.
} 
Canon) consisting of $5184 \times 3456$ pixels and disposed vertically at the top of the experimental model is used to acquire image sequences of viscous fingering at an image capture rate of 25 images/s. During data acquisition, the Hele-Shaw cell was illuminated by a homogeneous backlight. Preliminary tests have been performed to assess the maximal radial extent of fingering pattern beyond which the rectangular shape of the cell disturbs the finger propagation and generates an anisotropy. A value of $80 \mathrm{~mm}$ has been obtained.

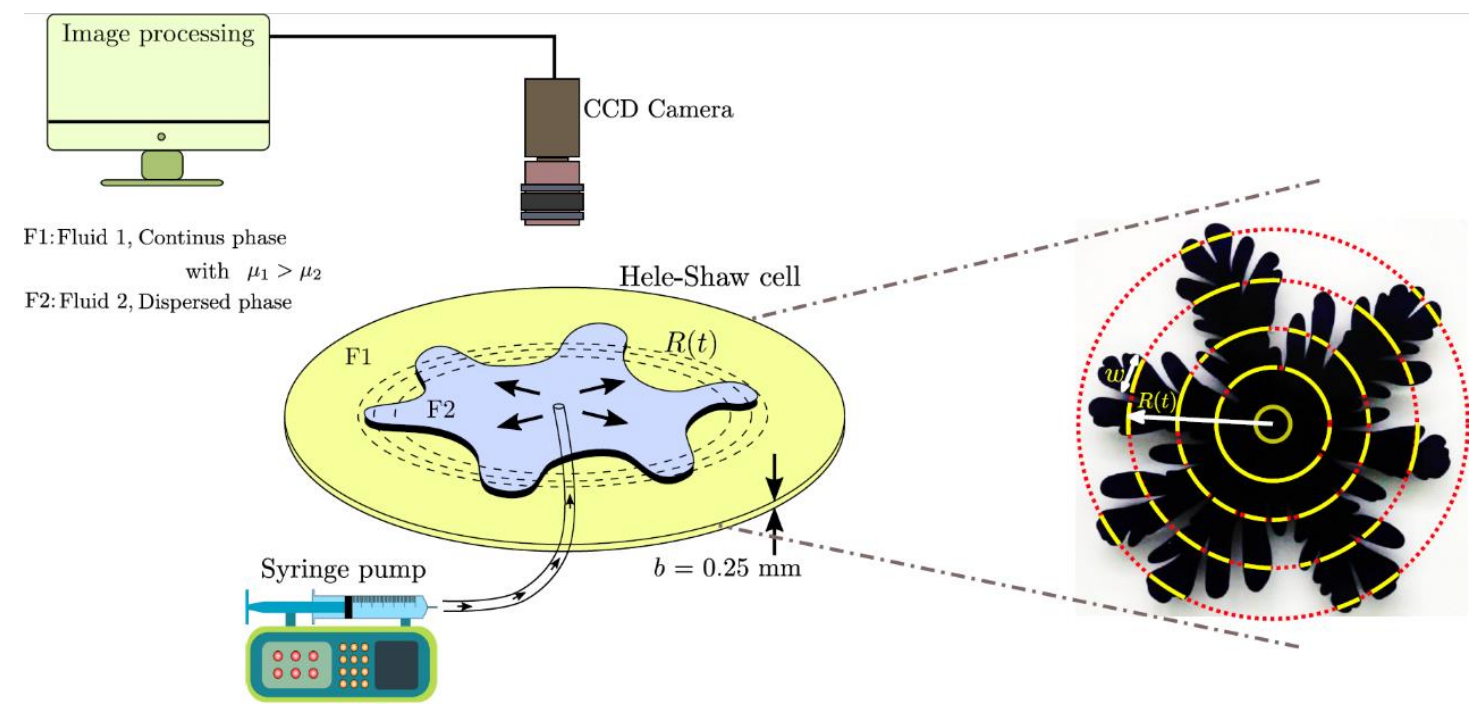

FIG 3. Radial viscous fingering experiment. (a) a schematic view of experimental set-up. (b) definition of the characteristic length scales

$$
R(t), w
$$

\section{Image analysis and processing}

The recording images were analyzed by using the Image ${ }^{\circledR}$ software package. To obtain quantitative analysis from images, the color pictures were first converted into binary images in Image J. We measured the number of fingers $(N)$ and the average finger width $(W)$ as a function of the distance $R(t)$ from the injection point using a classical procedure based on the intersection between the finger tips and concentric circles centered on the injection point (Fig. 3b). Then, we determined the finger propagation velocities $V_{f}$ defined as the ratio between the position of the measured finger tip relative to injection point and the travel time. The pressure gradient along the finger is estimated from the analytical solution of Herschel-Bulkley fluid flow between two plates ${ }^{47}$ (see also Appendix A for detailed calculations). The local apparent viscosity could be calculated in a similar way but the question of the representative mean value of $\mu$ to be used in our study remains open. The apparent viscosity, indeed, vary locally from a finite value at the channel walls to infinity in the middle of the flowcell. To remain consistent with the Hele-Shaw analogy with a porous medium, we define an effective viscosity $\mu_{e f f}$ obtained from the Darcy law and using the pressure gradient value previously calculated,

$$
\mu_{e f f}=\frac{b^{2}}{12} \frac{1}{V_{f}} \frac{\partial P}{\partial x}
$$

Finally, the equivalent shear rate can be back-calculated from the $\mu_{e f f}-\dot{\gamma}$ relationship defined from the Herschel-Bulkley like model as the ratio of the shear stress to the strain rate:

$$
\mu_{e f f}(\dot{\gamma})=\tau_{0} \dot{\gamma}^{-1}+K \dot{\gamma}^{n-1}
$$


Based on these data, a modified definition of the capillary number $\left(\mathrm{Ca}^{*}\right)$ representative of our experiments is used, where $V_{f}$ is the characteristic velocity and the non-Newtonian behavior has been taken into account by replacing the dynamic viscosity of the invading fluid $\mu$ by the effective viscosity $\mu_{e f f}$,

$$
C a^{*}=\frac{v_{f} \Delta \mu_{e f f}}{\sigma}
$$

The relative finger width $(\lambda)$ is also calculated and defined as follows ${ }^{37}$ :

$$
\lambda=\frac{\theta_{i}(t)}{\theta_{0}}
$$

where $\theta_{0}$ represents the initial angular width of a single finger (see fig. 8a) at the very start of the fingering pattern and $\theta_{i}$ the angular width of the same finger measured at the next time sequences $(\delta R)$. For a given pattern, the final value of $\lambda$ is averaged over all the counted fingers.

\section{RESULTS AND DISCUSSION}

Three sets of experiments have been carried out under different flow rates to examine the rheological and interfacial properties governing viscous fingering in radial Hele Shaw cell. The first describes the classical Saffman-Taylor instability for immiscible Newtonian fluids with a glycerol/silicone oil system and will be used hereafter as a reference pattern set. The second set of experiments corresponds to the injection of non-Newtonian shear thinning liquid (Carbopol) into silicone oil in the absence of surfactant. Finally, in the last series of experiments, the behavior of a surfactant (SDS)-polymer (Carbopol) mixture is investigated.

The range of flow rates and shear rates under investigation (with the corresponding effective viscosity of the injected fluid) for these three systems is shown in Table II. The values of capillary number $\mathrm{Ca}$ are also given. All the flow rates are sufficiently low to ensure a laminar flow. Each experiment was run three times in order to test the reproducibility of the results. In the further figures, all the reported values (e.g., finger length and width, maximal number of fingers) are calculated from the mean of triplicate experiments. The measured standard deviation from the repeatability study is about less than $10 \%$.

TABLE II. Range of experimental data

\begin{tabular}{ccccc}
\hline \hline Samples & Flow rate & $\dot{\gamma}$ & $\mu_{\text {eff }}$ (displacing fluid) & $C^{*}$ \\
& $m L . h^{-1}$ & $s^{-1}$ & Pa.s & \\
\hline Glycerol / silicone oil & $3.5-10$ & $7.68-17.54$ & 1.26 & $(0.57-1.31) \times 10^{-5}$ \\
Carb-SDS 0.0 / silicone oil & $3.5-10$ & $1.44-8.52$ & $1.76-7.57$ & $(1.5-2.16) \times 10^{-5}$ \\
Carb-SDS 0.01/ silicone oil & $3.5-10$ & $1.55-9.31$ & $1.56-6.76$ & $(2.68-3.89) \times 10^{-5}$ \\
\hline \hline
\end{tabular}

\section{A. Fingering patterns for Newtonian and non-Newtonian fluids}

Radial fingering patterns obtained from the different sets of experiments and for increasing values of flow rates are shown in Fig.4. Depending on the nature of the displacing fluid, two different behaviors are observed. For the classical ST instability 
(Fig.4a-c), pattern grows forming fingers by successive tip-splitting. When the flow rate increases, the interface becomes more unstable and the number of fingers increases. The fingers become narrower and side-branching occurs 3,4,5,8,14,20.
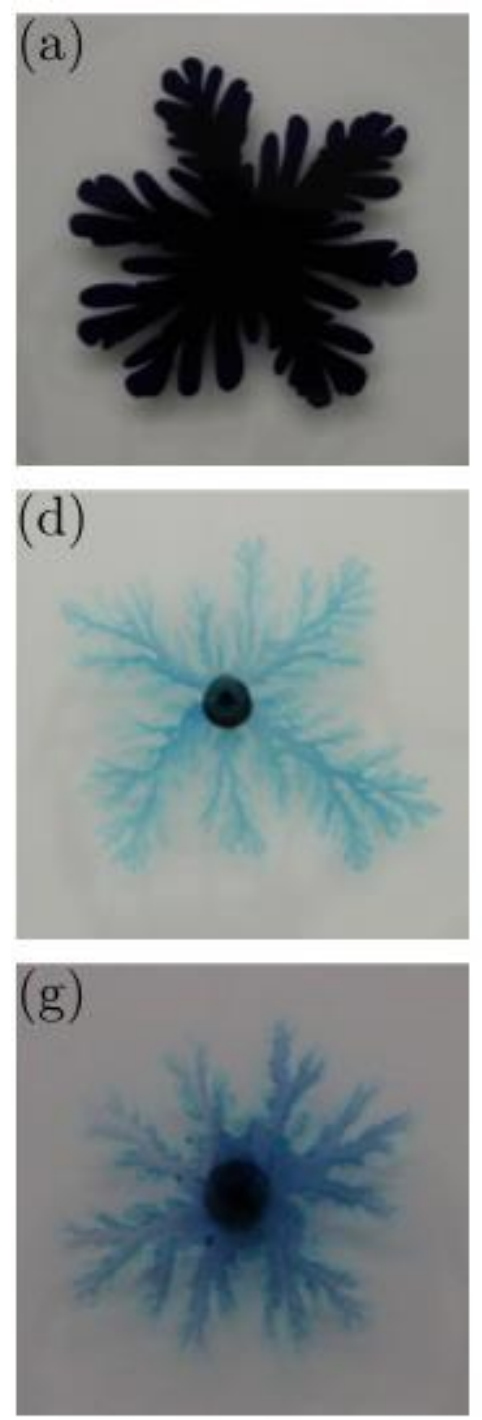
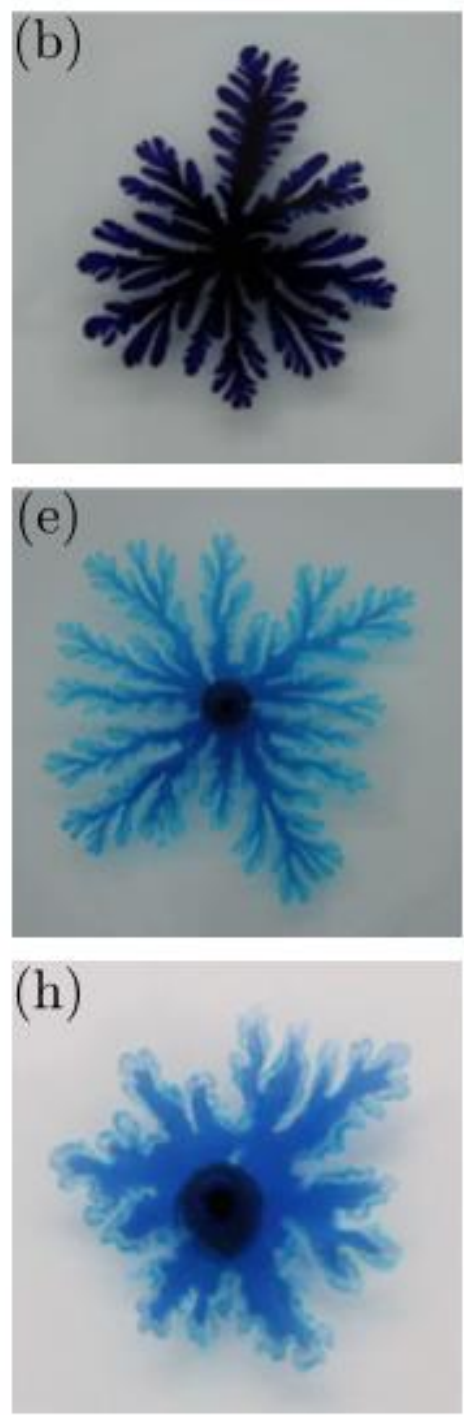
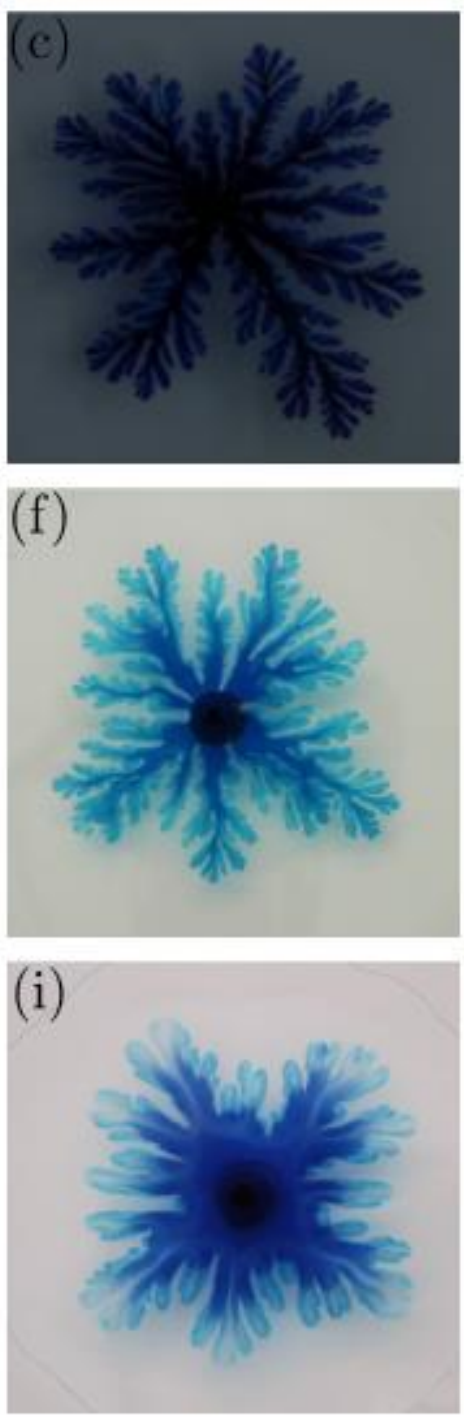

FIG. 4. Fingering patterns for different displacing fluids : Glycerol $(a, b, c)$, Carbopol $0.12 \%(d, e, f)$ and a mixed solution of Carbopol 0.12\% $+\operatorname{SDS} 0.01 \%(g, h, i)$ and various flow rates: $3,5 \mathrm{~mL} / \mathrm{h}(a, d, g), 7 \mathrm{~mL} / \mathrm{h}(b, e, h)$ and $10 \mathrm{~mL} / \mathrm{h}(c, f, i)$.

Our observations are significantly different in the both cases of Carbopol injection with and without surfactant, (Figs. 4d-f and 4g-i). For pure Carbopol solution, fingering pattern is very branched and narrow at low flow rate (Fig. 4d). However, the increase in the flow rate leads to the formation of fewer and wider fingers (Fig. 4e,f). Surfactant addition results in wider fingers (Fig. 4g) but similar behavior is obtained relative to the flow rate (Fig. 4h,i).

In order to get a deeper understanding on the role of fluid rheology and surface tension on viscous fingering, changes in finger number $(N)$ and width $(\bar{w})$ as a function of distance $(R(t))$ from injection point are illustrated in Fig.5. This figure (Fig.5a-c) reveals for all the experiments an increase of the number of fingers until a certain threshold value and then a decrease due to shielding effect ${ }^{38,39}$. Initially, the fingers split successively into more fingers but after a certain time, some of them become predominant and shield the adjacent fingers from further growth. At low flow rate, the number of fingers is initially higher for the polymer than for Glycerol due to the higher polymer viscosity that tends to destabilize the flow but the opposite trends in 
terms of flow rate effect lead to a reverse pattern at $Q=10 \mathrm{~mL} / \mathrm{h}$. In the last series of experiment, the modification of surface tension induced by adding surfactant yields always a reduction of the number of fingers and seems also to slightly delay the shielding effect.

An opposite but consistent behavior is found for the variations of finger width which decreases as the finger tip is far away from the injection source (Fig.5a'-c'). Basically, the more unstable the pattern is, the narrower the fingers are. However, we do not observe any extremum; the shape of the curve is more smoothed and converges toward a plateau even if the number of fingers is still decreasing. This result is consistent with the asymptotic value of the relative finger width predicted by the Saffman-Taylor theory ${ }^{3}$ (or by Bonn et al. ${ }^{13}$ for non-Newtonian fluids for instance). In spite of the shielding effect, the fingers do not continue to enlarge beyond a critical value but tend towards a solution linearly stable driven by the surface tension ${ }^{8}$. Regarding the flow rate dependency on finger width, results are consistent with those observed in terms of finger number as noted previously. Contrary to ST theory, finger width increases (resp. finger number decreases) with flow rate for both nonNewtonian fluids and this is in agreement with the literature ${ }^{8}$. This dependency of finger width with $V_{f}$ confirms that we are not in the yield stress regime but rather in the viscous regime identified by ${ }^{15}$. Note that Eslami et al. ${ }^{15,16}$ observe an opposite result in terms of finger width evolution for air/carbopol system but the displaced fluid is the non-Newtonian one so that mechanisms are not similar and comparison is pointless.
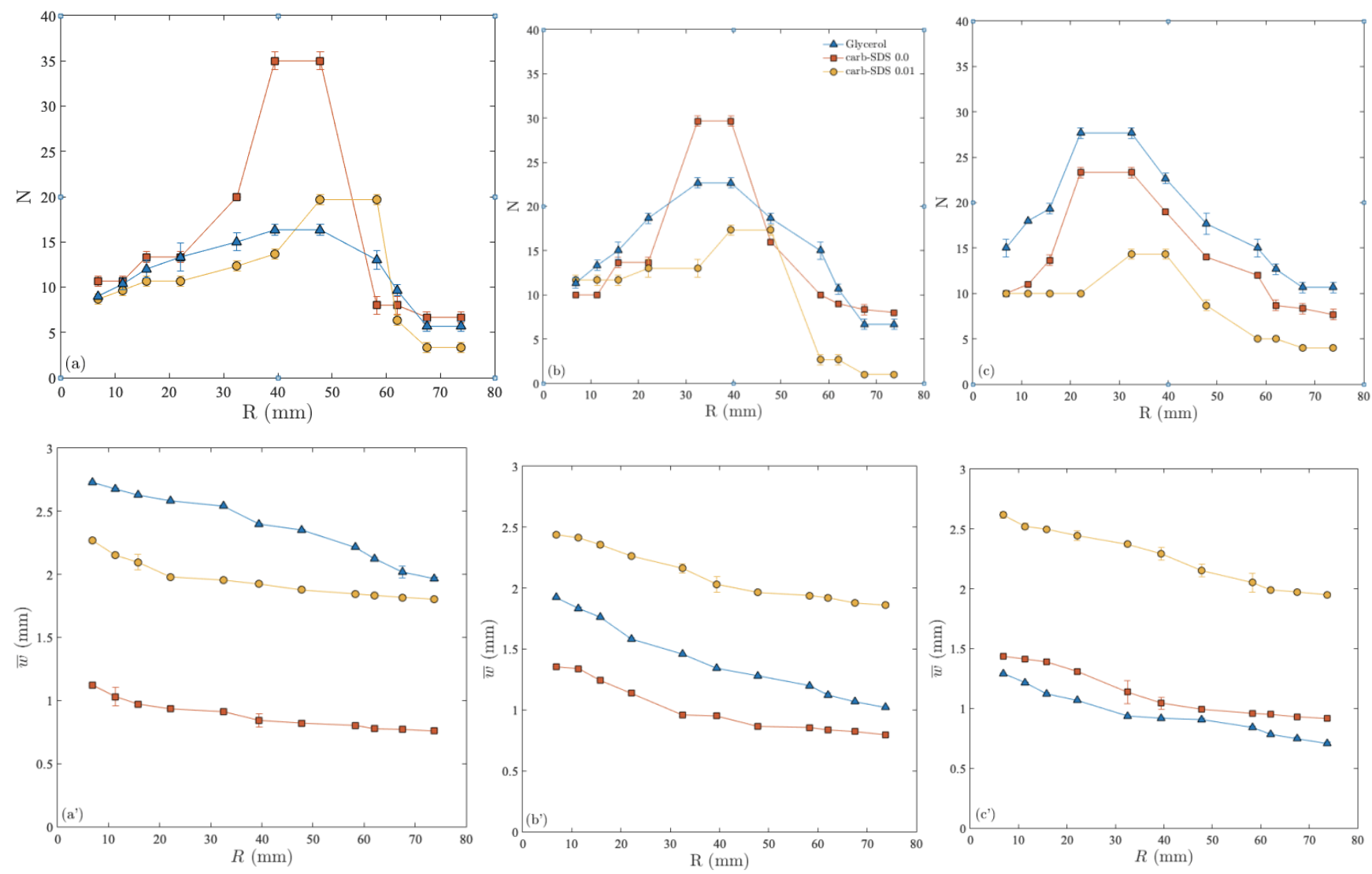

FIG. 5. Finger number and width variation as a function of distance from injection point for Newtonian and non-Newtonian fluids 


\section{B. Short-term and long-term effect of surfactant addition on capillary-number dependency}

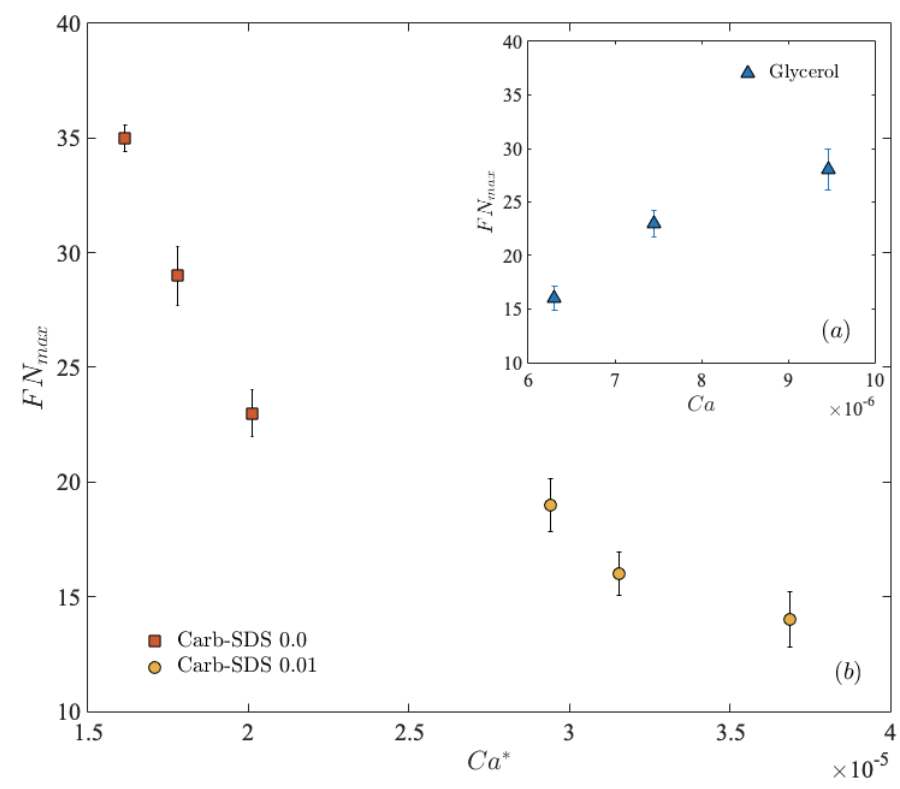

FIG. 6. Evolution of maximal number of fingers as a function of capillary number for a) Glycerol and b) Carbopol ${ }^{\circledR} 940$ and mixed Carbopol-SDS solution.

The discrepancy with the Saffman-Taylor theory for polymer and polymer-surfactant solutions is highlighted in Figure 6. We have represented here the maximal number of fingers $\left(F N_{\max }\right)$ measured for each experiment as a function of the capillary number. In the case of ST instability, the fingering pattern becomes more and more unstable (and hence the finger width decreases) with increasing capillary number (viscous forces tend to narrow the finger and capillary forces to widen it). The opposite effect is observed for the non-Newtonian fluids in the present work. Which rheological features of Carbopol and Carbopol-SDS are responsible for such dynamics? For pure polymer solution, this question has been already addressed by 9,13 and our results are consistent with their observations. They have found that for shear-thinning fluids such as Carbopol, narrower fingers are obtained due to shear rate variations and, hence, of viscosity, along the finger. As an illustrative purpose, we represented the changes in viscosity along one finger produced by Carbopol $0.12 \%$ injection in Figure 7 . Assuming constant flow rate within the finger and knowing the finger velocity $V_{f}$, we measured the finger width variations as a function of the distance $R(t)$ for a single finger at a given time and calculated the associated effective viscosity values within the finger (using Appendix A). Figure 7 points out a significant viscosity decrease of more than $30 \%$ along the finger which supports the assumptions of ${ }^{9,13}$. Note that Carbopol exhibits also weak elastic properties but this cannot be the cause of such behavior since the normal stress effects should rather tend to widen the fingers ${ }^{13}$. In addition, these effects can be neglected for the range of shear rate investigated. 


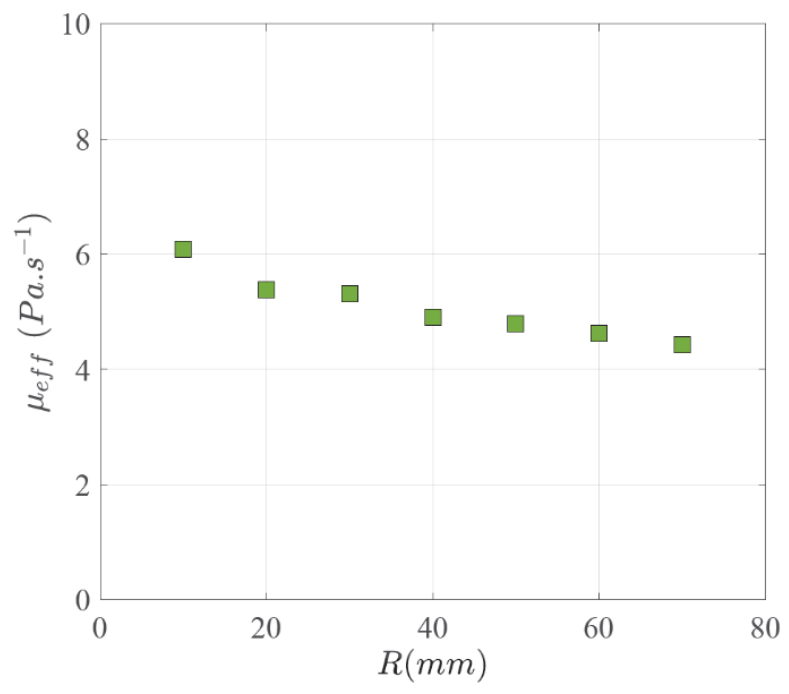

FIG. 7. Evaluation of the viscosity $\mu_{e f f}$ as a function of $R(t)$ for one finger produced from Carbopol $0.12 \%$ solution.

In the presence of surfactant, the evolution of $F N_{\max }$ is similar to the one of pure Carbopol solution but the $F N_{\max }$ value is lower. Contrarily what we could expect, the surface tension reduction which should tend to narrow the finger has an opposite effect. This unexpected impact is probably due to the advection of surfactant molecules along the finger surface and the resulting anisotropy of surface tension as suggested by ${ }^{8}$. This result is consistent with the observations of ${ }^{12}$ for surfactant-non Newtonian solution and ${ }^{19}$ for surfactant-Newtonian solution. However, the finger width decreases classically with increasing finger velocity in ${ }^{19}$ as for a pure Newtonian fluid. In other words, the surfactant induces always larger fingers because of the abovementioned mechanism but the flow rate dependency is driven by the Newtonian or non-Newtonian nature of the fluid.

Finally, we represent in Fig. 8 the variations of the relative finger width as a function of $\mathrm{Ca}^{*}$. Classically, $\lambda$ tends towards 0.5 for Glycerol-silicone in agreement with ST theory ${ }^{3,4}$. On the contrary, both non-Newtonian solutions converge to a value a little bit lower than 0.5, about 0.4. If this behavior is in agreement with the state of the art for pure Carbopol solution ${ }^{8,9}$, the result is more surprising for two reasons when adding surfactant. First, ${ }^{8,13}$ pointed out a relative finger width above 0.5 for oilwater-surfactant system but as previously, this discrepancy has to be put down to the non-Newtonian features of our polymersurfactant solution in the present work. In addition, this result could also seem contradictory - at the first sight - with the observations of Figs. 5 and 6 where differences in term of finger width between polymer and polymer-surfactant mixture were clearly visible. Actually, the average finger width $\bar{w}$ and the relative finger width $\lambda$ do not reflect the same features. $\bar{w}$ characterizes the changes in finger width induced by competition between instabilities in the radial fingering pattern whereas $\lambda$ represents the evolution with time of a single finger. In other words, $\bar{w}$ and $\lambda$ bespeak respectively the short-term and longterm (asymptotic) behavior of viscous fingers.

In light of the above observations, we propose the following explanation for the finger dynamics, consistent with the assumption of ${ }^{8,12,13}$ relative to the surface tension anisotropy. At the very start, surfactant injection affects fingering competition and tends to produce fewer and wider fingers. Then, as the instability is growing, surfactant molecules are flushed away from the tip to the base of the finger, whether or not compensated to some extent by Marangoni effect as suggested by ${ }^{19}$. As a result, this mechanism induces an increase of the interfacial tension in the vicinity of the finger tip. Ultimately, as the finger tip is propagating away from the injection source, the shear-thinning fluid behavior prevails and we recover the same relative finger width than for pure Carbopol. 


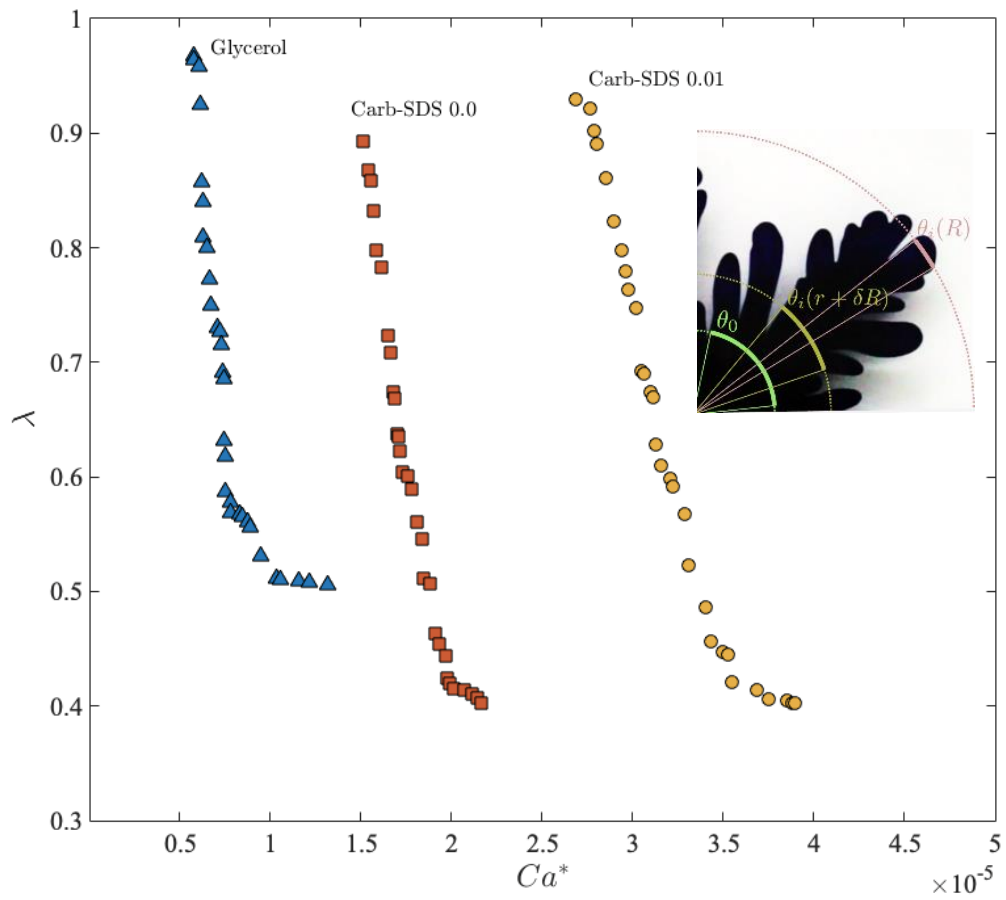

FIG. 8. The dimensionless relative finger width variation as a function of capillary number for Glycerol, Carbopol and mixed CarbopolSDS solutions.

\section{CONCLUSIONS}

We investigated the impact of surfactant addition on polymer injection into viscous oil with an emphasis on viscous fingering. We performed an experimental study in radial Hele-Shaw cell for deciphering and analyzing the driving processes in viscousfingering patterns resulting from different sets of fluids. Newtonian and non-Newtonian liquids were used as displacing fluids. As Newtonian fluid of reference we used Glycerol and for non-Newtonian fluids a pure polymer (Carbopol $\left.{ }^{\circledR} 940\right)$ and a mixed solution of polymer-surfactant (Carbopol-SDS). An estimate of the local pressure drop and effective viscosity was proposed based on the analytical solution of Herschel-Bulkley fluid flow in channel to rigorously analyze the fate of fingering patterns. Contrarily to what is predicted for Saffman-Taylor instability where the combination of viscous polymer and surfactant is expected to promote the oil displacement by both increasing the viscous forces and reducing the capillary forces, specific rheological features (shear-thinning fluid, surface tension anisotropy) modify this behavior. We thus pointed out that if surfactant concentration decreases logically the interfacial tension, the effect on $\mathrm{Ca}^{*}$ is enhanced to some extent by a reduction of viscosity due to an increase in the ionic strength of the medium ${ }^{40}$.

Regarding pure Carbopol solution, our observations are consistent with previous experimental evidence in viscous fingering. Shear-thinning features displayed by Carbopol result in a gradient of viscosity along the finger and make narrower fingers. In the presence of surfactant, experimental results are still in agreement with literature - surfactant injection leads to wider fingers due to surface tension anisotropy along the finger surface ${ }^{8,13,19}$ but with some distinguishing and original features. Two main results should be highlighted: 
- The flow rate dependency of surfactant solution behavior is driven by the Newtonian or non-Newtonian nature of the fluid. Surfactant-polymer solutions have wider fingers with increasing flow rates in contrast with surfactant-Newtonian solutions.

- The asymptotic behavior tends to smooth out the surfactant effect on fingering pattern. For both non-Newtonian fluids, the relative finger width converges practically towards the same value that is 0.4 at large $\mathrm{Ca}^{*}$. We assume that this phenomenon is caused by the decrease of surfactant concentration in the vicinity of the tip as the finger is growing.

Ultimately, since the Hele Shaw cell can be seen as an idealized model of fracture, a more significant insight into the behavior of surfactant-polymer solutions in fractures should be obtained from this work.

\section{Appendix A: Analytical solution of Herschel-Bulkley fluid flow between two parallel plates}

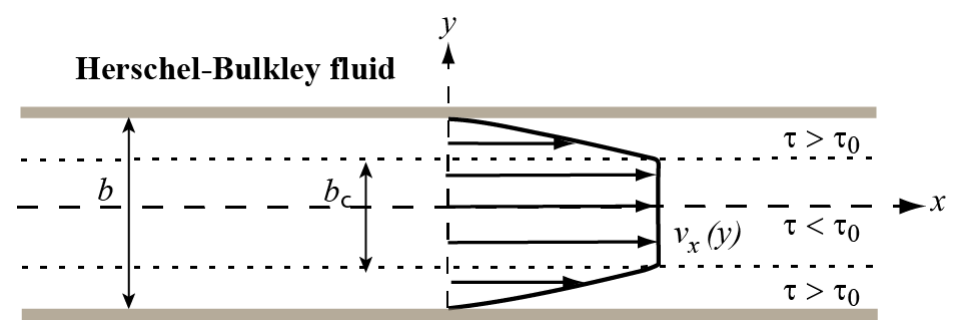

FIG. 9. Herschel-Bulkley fluid flow between two plates

We consider a Herschel-Bulkley fluid flowing steadily between two fixed horizontal parallel plates as illustrated in Fig. 9. The plates are a distance $b$ apart from each other and the coordinate system is centered in the middle of this distance so that the top plate is located at $\mathrm{y}=b / 2$ and the bottom plate is located at $\mathrm{y}=-b / 2$. We postulate laminar and incompressible flow between plates. A constant pressure gradient is imposed across the channel, i.e., $\partial P / \partial x>0$. Additionally, the pressure is assumed to be constant over the cross-section of the channel, i.e., $\partial P / \partial y=0$. A no-slip boundary condition is imposed at the walls such as:

$$
v(y)=0 \text { at } y= \pm \frac{b}{2}
$$

The three-parameter Herschel-Bulkley fluid model can be written as follows:

$$
\begin{cases}\dot{\gamma}=0 & \text { if } \tau \leq \tau_{0} \\ \tau=\tau_{0}+K \dot{\gamma}^{n} & \text { if } \tau>\tau_{0}\end{cases}
$$

Starting with the momentum balance equation, we have

$$
\tau_{x y}=\frac{\partial P}{\partial x} y
$$

Using the constitutive relation for Herschel-Bulkley fluid, we may define a critical width $b_{c}$, above which the threshold yield stress is reached ${ }^{41}$, such as

$$
\frac{b_{c}}{2}=\frac{\tau_{0}}{\frac{\partial P}{\partial x}}
$$

An expression for the velocity profile is obtained by rearranging the rheological model and integrating the strain rate over the channel. Given the symmetry of the solution, the problem is solved in half of the domain, where $y>0$. After calculation, we obtain 


$$
\left\{\begin{array}{lr}
y \in\left[0, \frac{b_{c}}{2}\right] & v_{x}(y)=v_{\max }=\frac{n}{n+1}\left(\frac{1}{K} \frac{\partial P}{\partial x}\right)^{\frac{1}{n}}\left(\frac{b-b_{c}}{2}\right)^{1+\frac{1}{n}} \\
y \in\left[\frac{b_{c}}{2}, \frac{b}{2}\right] & v_{x}(y)=v_{\max }\left(1-\left(\frac{2 y-b_{c}}{b-b_{c}}\right)^{1+\frac{1}{n}}\right)
\end{array}\right.
$$

The average velocity is given by

$$
V=v_{\text {moy }}=\frac{2}{b} \int_{0}^{b / 2} v_{x}(y) d y
$$

The solution depends on the value of the channel width $b$ relative to $b_{c}$ and a straightforward calculation leads to

$$
\left\{\begin{array}{rr}
b \geq b_{c} & V=\frac{2 v_{\max }}{b(2 n+1)}\left[\frac{b}{2}(n+1)+\frac{b_{c}}{2} n\right] \\
b \leq b_{c} & V=0
\end{array}\right.
$$

Note that for $\mathrm{n}=1$ we recover the literal expression for a Newtonian fluid.

Given the mean velocity $V$, estimated from the finger velocity $V_{f}$, and from the rheological parameters in Table I, the above equation can be solved for the pressure loss by a simple iterative technique. At this point, $b_{c}, \dot{\gamma}(y)$ and the apparent dynamic viscosity $\mu(y)$ could be determined locally in any point along the cell aperture. To remain consistent with the analogy of $2 \mathrm{D}$ porous medium, we prefer define an effective viscosity $\mu_{e f f}$ based on the Darcy law expressed as:

$$
\mu_{e f f}=\frac{b^{2}}{12} \frac{1}{V_{f}} \frac{\partial P}{\partial x}
$$

\section{REFERENCES}

${ }^{1}$ A. Muggeridge and A. Cockin, "Recovery rates, enhanced oil recovery, technological limits," Math Phys Eng Science 372 (2014).

${ }^{2}$ P. Raffa, A. Broekhuis, F. Picchioni, "Polymeric surfactants for enhanced oil recovery: A review," Journal of Petroleum Science and Engineering 723-733, (2016).

${ }^{3}$ P. G. Saffman, “Viscous fingering in Hele-Shaw cells,” J. Fluid Mech 173, 73 (1986).

${ }^{4}$ Homsy, G.M, “Viscous fingering in porous media.” Annu. Rev. Fluid Mech 271-311, 19,1 (1987).

${ }^{5}$ E. Lajeunesse, J.Martin, N. Rakotomalala, D.Salin. “3d instability of miscible displacement in Hele-Shaw cell,”

Phys.Rev.Lett 5254-57, 79 (1997).

${ }^{6}$ R. Maes, G. Rousseaux, B. Scheid, M. Mishra, P. Colinet, and A. De Wit, “Experimental study of dispersion and miscible viscous fingering of initially circular samples in Hele-Shaw cells," Physics of fluids 123104, 22 (2010).

${ }^{7}$ J. D’Hernoncourta, S. Kalliadasis, A. De Wit, “Fingering of exothermic reaction-diffusion fronts in Hele-Shaw cells with conducting walls," The journal of chemical physics 234503, 123 (2005).

${ }^{8}$ A. A. Lindner, D. Bonn, J. Meunier, “Viscous fingering in a shear-thinning fluid,” Phys. Fluids 256-261,12 (2000).

${ }^{9}$ A. Lindner , P. Coussot , D. Bonn , “Viscous fingering in a yield stress fluid,” Phys. Rev. Lett 314, 85 (2000).

${ }^{10}$ N. Kagei , D. Kanie , M. Kawaguchi , “Viscous fingering in shear thickening silica suspensions,” Phys. Fluids 0545103, 17 (2005) .

${ }^{11}$ T. Yamamoto, Y. Nakamura, A. Yamashita, T. Hashimoto, N. Mori "Anomalous motion of viscous fingers in surfactant solutions in a Hele-Shaw cell," Rheol Acta 250-259, 45 (2006).

${ }^{12}$ D. Bonn , H. Kellay, J. Meunier, “Viscous fingering and related instabilities in complex fluids,” Philosophical Magazine B 131-142, 78:2 (1998). 
${ }^{13}$ D. Bonn, H. Kellay, M. Ben Amar, J. Meunier, "Viscous finger widening with surfactants and polymers,” Phys. Rev. Lett 2132, 75 (1995).

${ }^{14}$ C.Y. Chen, P.Y. Yan, "Radial flows in heterogeneous porous media with a linear injection scheme," Computers and Fluids 30-36, 142 (2017) .

15 A. Eslami, S.M. Taghavi, "Viscous fingering regimes in elasto-visco-plastic fluids, " Journal of Non-Newtonian Fluid Mechanics 79-94, 243 (2017).

${ }^{16}$ A. Eslami, S.M. Taghavi, "Viscous fingering of yield stress fluids: The effects of wettability, " Journal of Non-Newtonian Fluid Mechanics 25 - 47, 264 (2019).

${ }^{17}$ A. Abbasi Yazdi, M. Norouzi, "Numerical study of Saffman-Taylor instability in immiscible nonlinear viscoelastic flows," Rheologica Acta August, (2018).

18 M. Sastry, A. Gole, A. G. Banpurkar, A. V. Limaye, and S. B. Ogale, "Variation in viscous fingering pattern morphology due to surfactant-mediated interfacial recognition events," Curr. Science 81,191 (2001).

${ }^{19}$ R. Tsuzuki, R. Tanaka, T. Ban, Y. Nagatsu, "Deviation from capillary number scaling of nonlinear viscous fingering formed by the injection of Newtonian surfactant solution," Phys. Fluids. 042108, 31 (2019).

${ }^{20}$ Z. Niroobakhsh, M. Litman, A. Belmonte, "Flow instabilities due to the interfacial formation of surfactant-fatty acid material in a Hele-Shaw cell, " Physical review 053102, 96 (2017).

${ }^{21}$ Z. Niroobakhsh, A. Belmonte, "Dynamics of a reactive micellar oil-water interface in a flowing liquid column," Journal of Non-Newtonian Fluid Mechanics 111-122, 261 (2018).

${ }^{22}$ E. Goddard, "Polymer/Surfactant Interaction: Interfacial Aspects", Journal of Colloid and Interface Science 228-235, 256 (2002).

${ }^{23}$ B. Kronberg, K. Holmberg, B. Lindman, "Surface chemistry of Surfactants and Polymers, " Lisbon, Portugal, 2017.

${ }^{24}$ C. Wang and K. C. Tam, “New insights on the interaction mechanism within oppositely charged polymer/surfactant systems, "Langmuir 6484-6490, (2002).

${ }^{25}$ C. Zhang, H. Wen, Y. Huang, W. Shi, "Adsorption of anionic surfactants from aqueous solution by high content of primary amino cross-linked chitosan microspheres, " International journal of biological macromolecules 635-641, 97 (2017).

${ }^{26}$ J.S. Lee, K.W. Song, "Rheological Characterization of Carbopol 940 in Steady Shear and Start-up Flow Fields, " Annual Transactions of the Nordic Rheology Society 19 (2011).

${ }^{27}$ J.Y. Kim, J.Y. Song, E.J.Lee, S.K.Park, "Rheological properties and microstructures of Carbopol gel network system," Colloid Polymer Science 614-623, 281 (2003).

${ }^{28}$ C. Wang, C. Tam, “Interactions between Poly (acrylic acid) and Sodium Dodecyl Sulfate: Isothermal Titration, Calorimetric and Surfactant Ion-Selective Electrode Studies, " J. Phys. Chem. B 5156-5161, 109 (2005).

${ }^{29}$ R. Miller, E. V. Aksenenko, V. B. Fainerman, "Dynamic interfacial tension of surfactant solutions," Advances in Colloid and Interface Science 115-129, 247 (2017).

${ }^{30}$ S. Javadian, J.Kakemam, "Intermicellar interaction in surfactant solutions, a review study, " Journal of Molecular Liquids 115-128, 242 (2017).

${ }^{31}$ M. Milanović, "Insight into the Interaction Between Carbopol 940 and Ionic/Nonionic Surfactant, " J Surfact Deterg 505516, (2015).

${ }^{32}$ D.Bo. AG, L. R, Felippe AC, D.Zanette, Minatti, "Association of anionic surfactant mixed micelles with hydrophobically modified ethyl(hydroxyethyl) cellulose," Colloids Surf 100-106, 380 (2011). 
${ }^{33}$ S. A. Bahrani, and C. Nouar, "Intermittency in the Transition to Turbulence for a Shear- Thinning Fluid in Hagen-Poiseuille Flow," J. Appl. Fluid Mech. 1-6, 7 (2014).

${ }^{34}$ A. M. V. Putz, T. L. Burghelea, "The solid-fluid transition in a yield stress thinning physical gel," Rheol Acta 673-689, 48 (2009).

${ }^{35}$ J. Y. Kim , J. Y. Song, E. J. Lee, S. K. Park, "Rheological properties and microstructures of Carbopol gel network system, " Colloid. Polym. Sci. 614-623, 281 (2003).

${ }^{36} \mathrm{C}$ Ferrera, J M Montanero and M G Cabezas, "An analysis of the sensitivity of pendant drops and liquid bridges to measure the interfacial tension," Meas. Sci. Technol 3713-3723, 18 (2007).

${ }^{37}$ H. Luo, M. Delshad, G.A. Pope, K.K. Mohanty, "Scaling up the interplay of fingering and channeling for unstable water/ polymer floods in viscous-oil reservoirs," Journal of Petroleum Science and Engineering 332-346, 165 (2018).

${ }^{38}$ H. Li, B. Maini, J. Azaiez, "Experimental and numerical analysis of the viscous fingering instability of shear-thinning fluids, " Can. J. Chem. Eng. 52-62, 84 (2006).

${ }^{39}$ W.B. Zimmerman, G.M. Homsy, "Viscous fingering in miscible displacements: unification of effects of viscosity contrast, anisotropic dispersion, and velocity dependence of dispersion on nonlinear finger propagation, " Phys. Fluids. 2348-2359, 4 (1991).

${ }^{40}$ R. Barreiro-Iglesias, C. Alvarez-Lorenzo, A. Concheiro., "Poly(acrylic acid) microgels (carbopol® 934)/ surfactant interactions in aqueous media. Part II: Ionic surfactants," International Journal of Pharmaceutics 179-191, 258 (2003).

${ }^{41}$ A. Islam, S. Chevalier, I. Salem, Y. Bernabe, R. Juanes, M. Sassi , “Characterization of the crossover from capillary invasion to viscous fingering to fracturing during drainage in a vertical 2D porous medium," International Journal of Multiphase Flow 279-291, 58 (2014).

${ }^{42}$ S. Berg, S. Oedai, D. W. van Batenburg, K. Elewaut, D. M. Boersma, E. M. Ineke, "Visualization of ASP core flood experiments with X-ray CT imaging", EAGE 18th European Symposium on Improved Oil Recovery, paper B12, Dresden, (2015).

${ }^{43}$ P. Daripa, G. Paşa, « On Capillary Slowdown of Viscous Fingering in Immiscible Displacement in Porous Media”,Transport in Porous Media, 1-16, 75(1) (2008)

${ }^{44}$ S. Berg, H. Ott, "Stability of CO2-brine immiscible displacement", International Journal of Greenhouse Gas Control, 188203,11 (2012).

${ }^{45}$ B. Zhao, C. MacMinn, B.K. Primkulov, Y. Chen, A.J. Valocchi, J. Zhao, ... R. Juanes, “Comprehensive comparison of porescale models for multiphase flow in porous media", Proceedings of the National Academy of Sciences of the United States of America, 13799-13806, 116(28), [201901619] (2019).

${ }^{46}$ S. Berg, "Marangoni-driven spreading along liquid-liquid interfaces", Physics of Fluids 21, 032105 (2009).

${ }^{47}$ P. Coussot, "Steady, laminar, flow of concentrated mud suspensions in open channel”, Journal of Hydraulic Research, 535559, 32(4) (1994). 